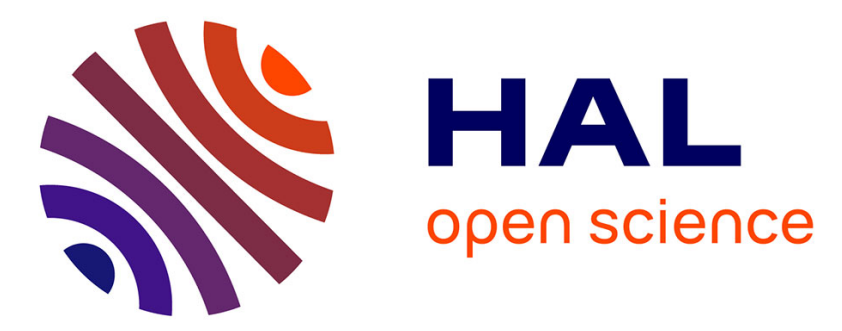

\title{
La Maison d'un artiste: fabrication textuelle d'une boîte à images
}

Dominique Pety

\section{To cite this version:}

Dominique Pety. La Maison d'un artiste: fabrication textuelle d'une boîte à images. Cahiers Edmond et Jules de Goncourt, 2004, Les Goncourt et l'image, 11, pp.145-158. 10.3406/cejdg.2004.934 . hal02464875

\section{HAL Id: hal-02464875 \\ https://hal.univ-smb.fr/hal-02464875}

Submitted on 3 Feb 2020

HAL is a multi-disciplinary open access archive for the deposit and dissemination of scientific research documents, whether they are published or not. The documents may come from teaching and research institutions in France or abroad, or from public or private research centers.
L'archive ouverte pluridisciplinaire HAL, est destinée au dépôt et à la diffusion de documents scientifiques de niveau recherche, publiés ou non, émanant des établissements d'enseignement et de recherche français ou étrangers, des laboratoires publics ou privés.

\section{(이) $\$$}

Distributed under a Creative Commons Attribution - NonCommercial - NoDerivatives| 4.0 


\section{La Maison d'un artiste : fabrication textuelle d'une boîte à images}

Le préambule de La Maison d'un artiste' annonce un licu saturé d'images. Edmond de Goncourt présente d'abord l'apparence extérieure de sa maison, mais se focalise ensuite sur un détail visuel significatif :

[...] une maison portant encastré dans son balcon un profil lauré de Louis $\mathrm{XV}$, en bronze doré, qui a tout l'air d'être le médaillon, dont était décorée la tribune de la salle de musique de la salle à manger de Luciennes, représenté dans l'aquarelle de Moreau que l'on voit au Louvre.

L'image (ici une image en relief, sculptée, monochrome) est encastrée dans la maison. Elle fait signe vers d'autres images, de nature différente : d'abord le souvenir historique d'un lieu orné, la résidence de la Du Barry à Louveciennes ${ }^{2}$; puis l'aquarelle d'un artiste déterminé, c'est-à-dire cette fois une image dessinée et colorée, laquelle renvoie à son tour à ce grand réservoir d'images artistiques et de souvenirs historiques qu'est le musée du Louvre. En outre, cette image, qui attire le regard (méfiant) des promeneurs, est comparée à d'autres images-signes, l'affiche, l'enseigne : si l'on ne doit pas, selon Goncourt, en fairc de lecture politique (encore que...), elle est la marque historique et esthétique d'une demeure dédiée à l'art et au XVIII' siècle.

C'est pourquoi il peut être fructueux d'appliquer les outils récemment proposés par Philippe Hamon à une relecture de ce texte, absolument exemplaire de l'envahissement des images dans la réalité et dans la littérature du XIX ${ }^{c}$ siècle, et qui peut ainsi servir de référence pour l'analyse de divers genres essentiellement fondés sur la transposition littéraire d'images réelles : le récit de voyage ${ }^{3}$, le

1. Dijon, L'Échelle de Jacob, 2003, 2 vol. (réimpression de l'édirion originale, Charpentier, 1881, complétée d'un index des noms propres, et de deux introductions de D. Pety et C. Galantaris). La présente étude espère aussi compléter les analyses engagées dans Les Goncourt et la collection. De l'objet d'art à l'art d'écrire, Droz, 2003.

2. Le lieu a été précisément décrit dans La Du Barry, 1878 (chap. VIII). Edmond de Goncourt renvoie donc aussi à une "image à lire " qu'il a lui-même produite.

3. Notamment ceux qui sont explicitement oricntés vers une collecte d'images, préludant à la sélection des référents à protéger, comme les Notes de voyage (1835-1840) de l'Inspecteur Général des monuments historiques Mérimée (rééd. Adam Biro, 2003). 
compte rendu d'exposition, le catalogue (de musée ou de collection privée, qui répondent à des normes différentes ${ }^{4}$ ), les mémoires ${ }^{5}$, la chronique journalistique type visite à une personnalité dans son intérieur ${ }^{6}$, etc.

Derrière les images (image à voir, image à lire de la description, image mentale du souvenir ou du rêve, pour reprendre la typologie de P. Hamon), se profilent évidemment les choses, ces objets denses, concrets, tridimensionnels, participant aux circuits de l'usage, de l'échange, subissant l'usure du temps aussi, mais dotés en revanche d'une présence plus tangible que la représentation en surface, en mots, ou en esprit. Aussi le "profil lauré de Louis XV" (un objet aussi, mais qui fonctionne d'abord comme une image, c'est-à-dire moins par ce qu'il est, que par ce qu'il représente) désigne-t-il surtout un " nid plein de choses ", un espace qui s'ouvre sur une population d'objets (" des terres cuites, des bronzes, des dessins, des porcelaines "), hérités précisément d'une culture de l'objet (les collectionneurs du XVIII siècle, dont Madame de Pompadour et " tous les curieux et curiolets du temps").

Le collectionneur considère l'objet dans sa totalité (physique, technique, historique), mais dans la jouissance esthétique, c'est particulièrement l'apparence visuelle qui le sollicite. Comme le décorateur d'intérieur qui agence un espace, ce sont alors essentiellement des effets visuels, des images, qu'il valorise et exploite, et celles-ci n'ont pas besoin d'être figuratives (les taches de couleurs des poteries japonaises, par exemple). De ce fait, on peut s'attendre à

Les Goncourt ont eux-mêmes pratiqué la plupart de ces différents genres : le carnet de voyage (France-Algérie, 1849, dont la correspondance nous conserve des extraits; Italie [Notes sur l'Italie (1855-1856), éd. N. Laneyrie-Dagen et E. Launay, 1996; L'Italie d'hier (1894)] ; AllemagneHollande [Journal, septembre 1860 et 1861]); le compte rendu d'exposition (Salon de 1852, Exposition universelle de 1855, exposition de l'art du XVIII' siècle au boulevard des Italiens, 1860) ; le catalogue (Watteau, Prud'hon, et l'enrichissement des biographies dix-huitiémistes); les mémoires (le Journal, qui évoque la maison d'Auteuil dans son évolution chronologique, et bien d'autres d'intérieurs...). Il faudrait voir, en reprenant les voies d'analyse qui sont ici utilisées, comment les images sont non seulement décrites (de la sécheresse dénotative du cacalogue à la subjectivité affichée du compte rendu [public] ou des mémoires [privées]), mais aussi quel soin est apporté à leur mise en scène, à la théâtralisation du regard qui les découvre, et enfin comment se définit le texte dans ses rapports à l'image qu'il commente et à la transposition qu'il produit.

4. Voir notamment Journal (30 mai 1861) à propos d'un ouvrage du siècle passé : "On n'a pas idée d'un catalogue aussi peu renseigné, aussi sommaire, aussi incomplet, aussi mal fait. Le moindre travail de catalographie de notre temps est à mille pieds au-dessus pour la science et la recherche. L'histoire, décidément et dans ses moindres branches, ne commence qu'au XIX siècle. "

5. Celles notamment d'un autre grand amateur d'images comme Montesquiou (Les Pas effacés, 1923).

6. Voir notamment Journal, 6 juillet 1890 («Hier, il est venu chez moi le reporter Puech de L'Éclair à l'effet de me peindre dans mon intérieur [...] "), et l'article d'O. Nora, "La visite au grand écrivain ", dans Les Lieux de mémoire, t. 11, 1986.

7. On sait qu'Edmond de Goncourt pose en collectionneur, mais aussi en "inventeur d'intérieur " dans La Maison d'un artiste ("Petit Salon ", I, 25). 
ce que la structuration esthétique de la collection ${ }^{8}$ se retrouve dans un texte descriptif qui transforme l'effet visuel en image à lire. Mais une problématique de l'image dépasse de beaucoup la seule question de la collection, et incite à analyser non seulement comment le texte littéraire amplifie la transformation des objets en images, mais aussi comment il multiplie les contiguités et les interactions entre différents registres d'images, qui ne renvoient pas toutes à un référent matériel, à un objet. En d'autres termes, la trame verbale densifie considérablement le réseau d'images que déployait déjà le collectionneur dans sa maison, prolifération dont il faut étudier les moyens et dégager les effets, lesquels ont aussi, on le verra, un revers négatif.

\section{L'image en soi(e)}

La première pièce de la maison (et le premier chapitre du livre) évoque bien la finalité décorative de la plupart des pièces de la collection, et donc l'exploitation prioritaire de leur apparence visuelle, de leur image. Après une formule générale ("toutes sortes de choses voyantes et claquantes"), vient un type d'objet spécifique, les soies brodées du Japon ou foukousas, qui apparaît comme l'image de base de La Maison d'un artiste : la plus répandue ("la riche, la splendide, l'éclairante décoration des murs, du vestibule et un peu de toute la maison"), la plus commune ("la chatoyante couverture sous laquelle on a l'habitude, dans l'Empire du Lever du Soleil, d'envoyer tout présent quelconque, et le plus minime, fût-il même de deux œufs"), et en même temps la plus élaborée (elle correspond à " un art tout particulier au Japon et auquel l'Europe ne peut rien opposer "). En outre, il s'agit là d'un objet bidimensionnel, réductible à sa surface, qui correspond le mieux, comme le dessin ou le tableau (auxquels il est d'ailleurs comparé, I, 5, I, 11), au prototype de "l'image à voir ".

C'est pourquoi il fournira, dans ce chapitre inaugural, les bases d'une réflexion technique et théorique sur l'image. Est d'abord évoqué son mode de fabrication (et il en sera de même, par la suite, pour tous les types d' "images ", estampe, céramique, tapisserie, etc., ainsi que pour les petits objets, bronzes, ivoires, laques. Dans les autres cas, particulièrement le mobilier, il est symptomatique que ce soit l'ornementation de l'objet, c'est-à-dire son apparence visuelle, décorative, qui soit privilégiée). Est ensuite soulignée la fidélité mimétique des motifs reproduits, la proximité de ces images avec le réel (" des ongles faits d'une soie qui joue la corne, de vrais ongles" I, 7), en même temps que l'exhibition de leur vocation artistique, avec par exemple, l'emploi de l'or (I, 10 ), de sorte qu'on a là des images gagnantes sur les deux plans, mimétique et sémiotique, à la fois "plein[e]s de vie " et "d'un si grand style" (I, 6). Et la

8. Manifeste aussi dans les photographies de la maison d'Auteuil. 
réflexion sur le statut ambigu de toute représentation se prolonge à propos de ce "brillant trompe-l'œil», où "l'illusion " n'existe pas seulement entre le référent et le motif, mais encore au niveau de l'exécution, qui imite d'autres formes d'art (" un foukousa qui donne l'illusion d'un croquis d'artiste "I, 12), comme pour dire que la médiation est bien le principe fondateur de l'image.

Ce qui se met ainsi progressivement en place, c'est aussi tout un système d'images. Non seulement chaque image est le réservoir d'autres images, par l'imbrication des motifs représentés (comme ce foukousa brodé de coffrets et de coquilles, dans lesquelles apparaissent "de minuscules Japonaises dans des jardins roses" I, 8-9), mais les liens sont aussi transversaux, et rapprochent les images de possesseurs distincts (" $\mathrm{M}^{\text {me }}$ Auguste Sichel " "M. de Nittis ", I, 6), d'époques et de techniques différentes ("l'envers des parties brodées est absolument l'envers du travail des tapisseries des Gobelins ", I, 9), de formes d'art très variées (ciselure d'or, laque polychrome, I, 9 ; dessin et peinture, I, 10).

Le but est certes d'abord d'établir l'égale dignité artistique de ce large éventail d'images ("la broderie conçue et exécutée ainsi n'est plus de l'industrie, mais bien un peu de l'art ", I, 11), rapprochées des plus nobles d'entre elles, celles issues des arts plastiques (les foukousas tiennent " d'une manière intime au grand art du dessin ", I, 10). Mais au-delà de ces correspondances entre les arts, il semble que Goncourt cherche à construire l'unité d'un vaste réseau d'images, comme si le pouvoir de l'écriture se mesurait à sa capacité à établir ce continuum visuel par lequel l'écrivain manifeste son emprise sur les choses, les hommes, les lieux et les époques, fondus dans un même moule. Pouvoir beaucoup plus vaste que celui du seul collectionneur : il procède structurellement des mêmes effets, mais il n'est pas assujetti comme lui à la possession des référents, il n'a besoin que de leur nomination, de leur évocation dans un style marqué. En outre, au-delà du mutisme premier de l'objet (qui ne livre rien à qui ne sait le regarder), ce système d'images manifeste un travail, une élaboration, une transformation : ce qui est devenu image, c'est ce qu'on a su voir, c'est ce dont le prestige visuel a été dégagé, particulièrement par le texte (d'où l'abondance de détails et la subjectivité euphorique du texte qui prend en charge l'image). Un système d'images, c'est un monde constitué, maîtrisé, révélé, offert à la jouissance esthétique et narcissique, et gage de stabilité psychologique... pourvu qu'on sache en garder la maîtrise.

\footnotetext{
"Emboîtement qui est une composante structurelle essentielle de la collection, comme le foukousa, lui-même enveloppe pour les présents au Japon, le signale d'entrée de jeu.
} 


\section{Les lieux à images}

Avant d'approfondir le rôle de l'écriture, il faut rappeler celui que joue essentiellement l'espace. Le collectionneur, le décorateur d'intérieur configurent les lieux en vue d'une mise en scène des objets, de façon à exploiter au maximum leur capacité à " faire image ". J'ai analysé ailleurs la fonction et la démultiplication des cadres, des boîtes, des étagères, et celui aussi de dispositifs plus complexes: l'escalier transformé en galerie, le cabinet de toilette en cabinet d'optique, et la chambre en salle de spectacle ${ }^{10}$.

Ces mises en scènes, dont le collcctionneur fai l'expérience dans le quotidien, semblent simplement transcrites dans La Maison d'un artiste. Mais d'autres configurations spatiales, exhibant des "images à voir ", nécessitent plus nettement la médiation de l'écriture pour fonctionner à plein régime : c'est le texte qui peut fixer les images mentales se greffant sur les images à voir, et prétendre les doter des mêmes qualités plastiques.

Ainsi le cabinet des dessins occupant le petit salon inclut-il, entre l'évocation du décor (revêtement des murs, ornements des cadres), et le catalogue des dessins eux-mêmes, la description, puisée dans le souvenir, des anciennes boutiques et des vieux marchands. Petites scènes de rues (" ces cartons bâillants entr'ouverts à la porte de ses centaines d'échoppes [...]») ou d'intérieurs (" une chaise cassée, puis un carton [placé] dans une filtrée de jour venant de la porte de la rue entre-bâillée [...]", I, 36), et surtout profils des marchands (" pittoresques silhouettes ", I, 34), qui sont autant de croquis signés Goncourt, et dont la qualité plastique tient à la cohérence et à l'accentuation de certains détails visuels dans un dessin à grands traits : Blaisot, "toujours en cravate blanche, avec du jovial et du renarré sur la physionomie, et une seule dent dans la bouche" (cravate blanche qui revient ensuite sur fond noir dans un dernier souvenir de Goncourt) ; pour Mayor, c'est une "caricature " qui emprunte ses motifs au potager et se teinte de couleurs (" deux petits yeux noirs, assez semblables à des pépins de poire, et un nez qui était comme une gousse de piment"; puis le portrait s'anime de mouvements d'automate, et finit sur un autre rouge de légume, avec le nez passé de l'écarlate à l'aubergine) ; Guichar-

10. Voir aussi ce dispositif plus sommaire (dans une maison où c'est au contraire l'ordre qui préside), le "vestibule-atelier ": "Sur ce cuir, dans un désordre cherché, dans un pitroresque d'antichambre et d'atelier, toutes sortes de choses voyantes et claquantes [...], de brillants cuivres découpés, des poteries dorées, des broderies du Japon et encore des objets bizarres, inattendus, étonnants par leur originalité, leur exotisme [...]" (I, 4). Dans cette phrase, c'est surtout la syntaxe, qui juxtapose des objets disparates, et le lexique de l'éclat et de la surprise, qui veulent faire percevoir ce désordre construit. Mais l'ensemble du chapitre répond à une structure rigoureuse : introduction par focalisation progrcssive (description du fond, du dispositif d'atelier, évocation semi-détaillée de quelques objets orientaux), long développement sur les foukousas, et description d'un bas-relief de Clodion qui ferme le chapitre comme une vignette, un cul-de-lampe. 
dot, enfin, réduit à un seul œil inquisiteur. Préludant à ses dessins du XVIII siècle, Goncourt livre ici quelques esquisses de son cru, si bien que le cabinet est encore plus riche qu'il n'y paraît d'abord. La surabondance de dessins des années 1840-1850, qu'il évoquait d'abord sur un ton nostalgique, il concourt luimême à la reproduire avec cette multiplication d'images, à l'intérieur d'un espace textuel qui reste par ailleurs soigneusement compartimenté : le XIX siècle reste cantonné dans la préface, isolée du catalogue de dessins du XVIII siècle, et ses croquis se distinguent en outre par leur motif et par leur tonalité propre (chaos des boutiques, outrance ironique ou fantastique des figures).

Après ce cabinet mêlant dessins réels et croquis souvenirs, autre dispositif qui va permettre de tresser images à voir et images mentales, celui du "gardemeuble " " qui, comme son nom semble l'indiquer, assemble mobilier et mémoire: "ici, c'est le petit garde-meuble des plus heureuses trouvailles de ma jeunesse " (Grand Salon ", I, 151 sq.). La description procède dans ce chapitre de manière originale : alors qu'elle fait généralement le tour des murs d'une pièce (voir particulièrement le "Cabinet de travail" et le "Cabinet de l'Extrême-Orient "), processus qui souligne surtout les surfaces et l'agencement de chaque mur en "panneau ", ici elle commence au contraire ( $1^{\text {er paragraphe) }}$ par une distribution des divers objets dans l'espace (au mur / au plafond / en dessous / dans les angles / au milieu), dispositif plus approprié pour évoquer une profondeur et les objets qui s'y déploient. Après quoi, ceux-ci seront décrits plus en détail, mais dans un ordre apparemment aléatoire, qui vise en fait à conjuguer l'apparence réelle des choses avec les images du souvenir. La description des statuettes de Clodion débouche ainsi sur l'évocation d'autres figurines du même sculpteur, qui ont jadis échappé au collectionneur débutant, d'autant plus précieuses semble-t-il qu'elles appartiennent au passé le plus ancien (" toutefois ce n'est pas mon regret le plus énorme. / Je sortais du collège [...]"). Les objets présents et les objets absents, ramenés au même statut d'images à lire, semblent avoir ainsi le même degré d'existence, d'autant que l'écriture exalte la beauté presque immatérielle des sculptures possédées, lesquelles, qualifiées de "rêve ", de "joli presque immatériel ", rejoignent à leur tour l'évanescence du souvenir. La tapisserie au plafond de la pièce s'associe à cet idéal : elle figure elle-même un ciel, un " coin d'Olympe ", et conduira tout naturellement au paradis perdu de la vie commune avec le frère (" $A$ ce nom de Wail, qui revient sous ma plume, que de souvenirs! Et les heureuses séances passées avec mon frère, en ces longues pièces obscures, où je vois encore [...]"). L'apparence des objets réels se mêle ainsi aux images des souvenirs personnels, mais aussi des souvenirs historiques, quant à eux déjà réactivés antérieurement par l'écriture : "Ils sont semblables, ces deux petits meubles, au mobilier garnis-

11. Voir aussi l'usage métaphorique du terme dans le Journal, 10 septembre 1860,14 février 1888 et 25 mai 1895 . 
sant la chambre de la Reine à Versailles dont j'ai donné la description d'après l'inventaire des 28 et 30 brumaire [...]"). Et l'évocation des porcelaines de Sèvres, de "la manufacture de $\mathrm{M}^{\mathrm{me}}$ de Pompadour ", renvoie pour sa part à la monographie des deux frères sur la favorite.

Dernier dispositif enfin, qui associe aux images à voir des images mentales, celui de la chambre mortuaire qui est aussi une "chambre noire " ${ }^{12}$. La chambre où Jules de Goncourt est mort, au "Second Étage ", "demeurée telle qu'elle était au lendemain de sa mort " (II, 370), garde l'empreinte de ses derniers jours (" ce que gardent et vous font retrouver d'un mort bien-aimé les choses de sa chambre mortuaire"). Il n'y a pas de comparaison explicite avec la plaque photographique, mais le lieu est garni d'images en noir et blanc (l'eau-forte, le dessin), il est lui-même noir et blanc (obscurité / "grande table en bois blanc "), et le frère aîné s'y installe à dates choisies pour voir se redérouler les images du passé : "De certains anniversaires et des jours de tristesse [...], je monte dans cette chambre, je m'assoie dans le grand fauteuil près du lit vide [...] / je me donne la douloureuse jouissance de me ressouvenir./ Et je le revois [...] quand $[\ldots]$, et que $[\ldots]$, et que $[\ldots] "($ III, 370). C'est bien un spectacle dans un crâne : les rideaux du lit encadrent la scène où eut lieu l'agonie. Par ailleurs, parmi ces souvenirs, il en est où les gestes de Jules sont "la mimique de son existence vécue ", le "simulacre" de son activité d'écrivain, de sorte que le comparant explicite pour caractériser ces images morbides est finalement celui de la pantomime ${ }^{1.3}$. La mort, c'est quand le vivant devient à son tour image, et dans cette distanciation, ne conserve qu'une similitude d'apparence par rapport à son identité réelle. L'image (caricaturale) est ici désignée comme ce qui vide l'humain de son humanité. Mais c'est aussi ce qui l'enrichit d'un sens qui le transcende : le visage de Jules se fige finalement en un tableau, aux contours et aux couleurs improbables (teint enfumé et doré, "sourire indéfinissable de ses lèvres violettes", qui lui donnent "une ressemblance troublante avec une figure mystérieuse et non humaine du Vinci "). Cette image picturale à son tour disparaît, et le masque de mort devient une image symbolique, "le désolé regret de l'CEuvre interrompu ". Ainsi l'agonie de Jules, comprise par Edmond comme un silence forcé imposé à la voix du frère, mais douloureusement restée dans son propre souvenir sous forme d'images prégnantes, est-elle aussi matière à réflexion plus générale sur la persistance et l'effacement des images (celles du vi-

12. Ce dispositif fonctionne aussi ailleurs, sous une forme moins développée : les portraits de famille (I, 354 sq.), images plastiques, images figées, images limitées, appellent le souvenir, image mentale, image agrandic, image animée.

13. La proximité entre pantomime et photographie est soulignée par P. Hamon. La chambre mortuaire est aussi une chambre phonographe, un lieu qui restitue les voix, altćrćcs (la lecture hésitante, la parole incontrôlée), comme il restitue l'image, sous une forme outrée, caricaturée. Cetre déformation marque la distance qui déjà sépare le moribond du vivant qu'il a été. 
vant, celles du souvenir), sur leur pouvoir, sur la menace aussi qui leur est inhérente : dans le corps réduit à l'image apparaît la vie atrophiée, ou sublimée.

\section{Le texte et les images}

Avec ces différents dispositifs, on voit donc bien le rôle du texte dans la production et l'unification des images. Mais il reste à analyser le discours métapoétique par lequel le texte veut tantôt faire oublier sa médiation, tantôt souligner sa nécessité, pris dans un rapport complexe avec cet autre système sémiotique, l'image, qu'il tente tout à la fois de seconder et de dépasser, ayant aussi à définir sa position à l'égard d'un terme médian, l'espace, avec son organisation propre et ses composantes référentielles. L'intérieur du collectionneur-écrivain est bien d'abord un lieu qui fabrique des images, mais qui produit aussi du texte, et du texte qui s'expose (livres garnissant les murs, livres reliés du "Cabinet de travail »). L'espace génère donc à la fois texte et images; quant au texte, non seulement il donne les images du lieu, mais il le sert aussi dans sa fonction de production, de multiplication, et (on le verra ensuite) de régulation des images.

Un des enjeux de La Maison d'un artiste est de fabriquer un genre spécifique (c'est le projet exposé dans la préface) qui associe le texte et l'espace, le texte et les choses, dans le sillage de la description, mais en allant plus loin dans cette association, avec les choses supplantant presque l'homme dans son rôle de premier plan, et occupant presque exclusivement le texte.

Pour construire ce texte-espace, l'accent est d'abord mis sur le motif du seuil, qui permet d'associer deux registres, le littéraire ("Préface ", seuil textuel), et le spatial ("Vestibule", seuil physique); la préface est en outre doublée d'un "Préambule", seuil à la fois littéraire et spatial, selon qu'on s'en rapporte au sens courant ou à l'étymologie, qui fait coïncider l'entrée dans le livre et l'entrée dans la maison, mise en scène de façon détaillée (" La porte noire, [...], la porte ouverte, du bas de l'escalier, de l'entrée du vestibule, du seuil de la maison, le visiteur est accueilli $[\ldots] » I, 1)$.

Or ce seuil, on a vu qu'il est décrit en termes d'images ${ }^{14}$. De fait, pour le texte, l'un des moyens de dire l'espace, au-delà des analogies lexicales (ex. "préambule ") ou structurelles (chapitres du livre / pièces de la maison), c'est de le donner à voir (par la description). Il s'agit donc de produire des images à lire, mais en s'interrogeant constamment sur leur statut, et sur la capacité du lisible à rendre compte du visible, particulièrement tridimensionnel et spatialisé. Tantôt le texte veut faire oublier sa médiation, en donnant à la description écrite la forme d'une parole orale qui guide la visite, comme s'il ne faisait que doubler

14. Voir aussi les analyses de P. Hamon sur le frontispice, l'image seuil, op. cit., p. 245 sq. 
une vision directe, alors qu'il la remplace : «voici " (I, 7), "voyez ", « regardez " (I, 8). Ou bien il rappelle qu'il fait partager une expérience qui est bien de l'ordre de la vision (" une fusée de fleurs brodées [...] qui est bien la plus harmonieuse chose qu'il soit possible de voir »), mais dont le référent est ailleurs, dans un espace autre ("chez $\mathrm{M}^{\mathrm{mc}}$ Auguste Sichel»), et comme tel, inaccessible sans l'intervention du scripteur. Ce qui s'affirme alors, c'est le besoin du texte pour accéder aux objets prisonniers d'un lieu, et plus largement, d'un contexte culturel lointain ("Voilà des choses que je ne connais pas, il faut que je fasse un livre dessus ", I, 4) ; c'est le texte qui permet de pénétrer dans le lieu, et même dans les images, elles-mêmes figurations d'autres lieux ("Mais entrons dans une de ces maisons, à l'aide d'une chanson populaire " I, 228) : le texte ouvre, alors que l'espace enferme, et c'est le texte qui permettra d'accéder aux secrets de l'image. Même s'il avoue parfois son impuissance (par la prétérition : "Je ne sais comment c'est fait $[\ldots]$ " I, 9), c'est pour mieux souligner le prestige de ce qu'il peut révéler. La fascination pour les objets hybrides, mêlant texte et image (dans l'album japonais, dans les idéogrammes, écriture qui est elle-même une manière de dessin, I, 231), relève peut-être du désir d'une idéale proximité entre le moyen et la fin, le texte faisant à son tour image, sans le truchement du référent et de l'espace qui l'enserre. Rêve d'une image entièrement visualisée par le texte, ou d'un texte entièrement image, où le référent, en la possession du collectionneur, n'est plus que la caution extérieure apportée à la description de l'écrivain. Il écrit: "J'ai, coloriée [...] l'échancrure pittoresque d'un passage de sept grues"; " j'ai encore, jetées sur un fond maïs, deux grandes grues blanches $[\ldots]$ " $(\mathrm{I}, 6)$, mais l'on entend : "j'ai colorié / j'ai jeté... ". C'est bien lui le nouveau créateur.

Mais le rôle de l'écriture par rapport aux images ne s'arrête peut-être pas là. Dans le processus de création et de multiplication des images, il est essentiel de garder la possibilité d'un contrôle, et là encore, l'espace sera mis en défaut. Le lieu parfois ne peut suffire à contenir le débordement des images qu'il a générées (" Là, dans ce petit salon est la plus grande partie de mes dessins, qui couvrent encore les parois du grand salon, montent et descendent l'escalier, remplissent les cartons dans cette chambre et cette autre, et se répandent ainsi par toute la maison. "I, 28). Cette prolifération était certes accrue, on l'a vu, par le texte lui-même, qui a ajouté d'autres types d'images, et contribué à niveler leurs différences, produisant une uniformité source de vertige. Pression quantitative et nivellement qualitatif qui risquent de conduire à une sorte de folie de l'image dont Nerval avait déjà détaillé le processus.

Dans Sylvie en effet, le narrateur expliquait ainsi sa fascination pour l'actrice Aurélie: "C'est une image que je poursuis, rien de plus." (chap. I ; de fait, l'actrice est, par définition une femme image). Mais sur cette image théâtrale se surimposait l'image du souvenir, par l'intermédiaire d'images plastiques : " La ressemblance d'une figure oubliée depuis des années se dessinait désormais avec 
une netteté singulière; c'était un crayon estompé par le temps qui se faisait peinture, comme ces vieux croquis de maîtres admirés dans un musée, dont on retrouve ailleurs l'original éblouissant. "Au point qu'on ne sait plus où est l'authentique, où est la copie. L'assimilation est effacement des différences, même les plus marquées, et cette perte des repères conduit à la folie : "Aimer une religieuse sous la forme d'une actrice !.. et si c'était la même! - Il y a de quoi devenir fou ! C'est un entraînement fatal où l'inconnu vous attire comme le feu follet fuyant sur les joncs d'une eau morte..." (chap. III). C'est cet univers uniformisé par la multiplication et l'équivalence généralisée des images que dénonce Nerval ; mais en même temps, il souligne son potentiel poétique, cette surface miroitante qui parvient à enchanter la réalité, à moduler infiniment son apparence ("Ermenonville! [...] tu as perdu ta seule étoile, qui chatoyait pour moi d'un double éclat [...] ", chap. XIV), d'autant plus nuancée qu'elle avait été enrichie d'allusions littéraires, d'images à lire, précisément (les descriptions de Rousseau, les idylles de Gessner, les vers de Roucher...).

L'objectif doit donc être, non le renoncement aux images, mais leur exploitation maîtrisée par le texte. Goncourt est lui aussi bien conscient de la part de menace qu'elles comportent. Dans ses romans, les héros conduits à la folie sont des victimes de l'image, également incarnée par la femme-actrice (Marthe Demailly, "l'imagination personnifiée " de l'écrivain [chap. XXXVIII], qu'il met en scène dans un décor d'images [chap. XLI]), ou la femme-modèle (Manette Salomon). On peut considérer qu'en elles la femme (charnelle) se dévoile et dénonce l'inconsistance de l'apparence (esthétique) à laquelle elles ont d'abord été réduites; mais ce faisant, elles jouent aussi de ce double visage, elles exploitent la diversité d'images qui est la leur pour mieux tromper. En outre, le spécialiste de l'image qu'est le peintre Coriolis est conduit sous cette influence à une folie proprement visuelle, une sorte d'hallucination des couleurs ${ }^{15}$, comme s'il n'avait plus la maîtrise de ce qui est le fondement même de son art. C'est donc le médium qui a contribué au déploiement des images, qui doit aussi en garder le contrôle, en excluant notamment ce qui pourrait y faire obstacle : la femme, prolifération d'images générées non par l'art, mais par le corps. De fait, dès le préambule, la femme, comme objet du désir amoureux, est absente de $L a$ Maison d'un artiste, en vertu d'une sorte de transfert d'images : à la place de celles qu'elle suscite d'ordinaire (quand elle occupe "l'imagination de l'homme "), sont venues s'inscrire celles des objets d'art.

Cette précaution prise, il faut certes concevoir un espace capable d'endiguer les images, parallèlement aux dispositifs spatiaux qui étaient chargés de les susciter. De fait, il y a, au second étage, des pièces qui occultent au lieu d'exhiber, et gardent par exemple les dessins serrés dans des portefeuilles. Le texte fera de

15. Manette Salomon, chap. CLI. Voir l'intervention de P. Tortonese ("La folie de l'œeil : à propos de Manette Salomon" au colloque Goncourt de la BnF, décembre 2003 (Acres à paraître). 
même, lorsque le descripteur dit omettre volontairement certains objets, et opère lui-même la censure parfois réalisée par le lecteur qui " saute " les descriptions. Mais surtout, en certains points stratégiques, l'écrivain doit témoigner de sa capacité à faire et à défaire l'image, à la réduire après l'avoir démultipliée.

Le chapitre consacré aux estampes japonaises ("Escalier») est fondé sur le déploiement des images contenues dans les albums, eux-mêmes enfermés dans un coffre sur le palier ${ }^{6}$. En outre, le Japon est par excellence le pays des images à profusion ("Le nombre, l'abondance, la prodigalité de l'image, au Japon, dépasse tout ce qu'on peut imaginer "I, 232), celui où les images se prolongent sur plusieurs feuilles (trois, six, jusqu'à douze), celui dont lcs habitants ont "la passion de l'image"; en outre, la fabrication des estampes, d'une simplicité déconcertante et d'une qualité exceptionnelle, réalise ce qu'on pourrait appeler un " comble de l'image ". Néanmoins, après cette exaltation de l'image, s'opère une sorte de repliement sur des images minimales, qui assurent la clôture du chapitre. Il s'agit à la fois d'images de la plus grande qualité (" des impressions exceptionnelles "), mais aussi de format restreint, de tirage limité, sur lesquelles le tracé semble à peine visible (" les linéaments des figures, tracés d'une manière presque imperceptible, les donnent à voir dans une espèce d'effacement vaporeux ") ; le motif presque insignifiant semble symboliser une éthique de l'image, une frugalité, une modération dans son usage, une quintessence de l'image instituée en minimum vital pour une existence d'artiste : Cela est tout, et cette représentation d'art de si peu de chose suffit à l'artiste, comme suffisait à Chardin la peinture d'un verre d'eau à côté de deux prunes!" (I, 237).

En d'autres endroits de La Maison d'un artiste existent des dispositifs similaires. Certes, l'effacement des images dans la "Chambre à coucher" (retour de l'obscurité après la "pastorale galante" qui s'est jouée dans la tapisserie à la lueur du foyer, II, 203) semble relever d'une sorte de respiration essentielle de l'image : son mode d'être commande ces deux phases opposées d'apparition et de disparition, dont le meilleur exemple est peut-être la représentation théâtrale. Mais, déployé à plus grande échelle, et lié de façon très nette à l'activité d'écriture, ce processus participe de l'effort de maîtrise du texte sur son univers d'images. Ainsi, dans le passage du "Cabinet de l'Extrême-Orient" au "Boudoir", s'opère une réduction d'échelle, et une sélection d'images ("seulement trois objets de haut goût", réductibles en outre à une surface plate : foukousa, panneau de laque, tapis de soie), qui fait se refermer la profusion précédemment déployée dans le cabinet (énumération d'ivoires, de bronzes, de céramiques, d'objets en laque...), en une sorte d'austérité indispensable à l'écriture (" Mais l'excitation produite par le bibelot de lumière [...] j'ai besoin

16. Par le truchement de l'album, c'est lc livre dans sá réalité physique qui est lui-même présenté comme une boîte à image, qu'on ouvre ou qu'on referme à volonté. Les volumes superbement reliés et illustrés du "Cabinet de travail " en fourniront d'autres exemples. 
pour écrire de me trouver dans une pièce qui n'ait rien aux murs, et que j'aimerais toute nue et blanchie à la chaux " II, 349).

Autre exemple, le jardin, jardin de peintre impressionniste déclinant en série les saisons, autre lieu à produire des images donc, est aussi, outre un jardin de collectionneur rempli d'objets, un lieu mêlé de souvenirs littéraires : jardin poésie (" quoi que je ne soit pas encore assez Japonais pour attacher aux branches de l'arbre admiré un sonnet commémoratif [mais que fait-il d'autre dans ces dernières pages de La Maison d'un artiste ?], il m'arrive de demeurer un long temps à jouir de la vue [...]" II, 378), jardin théâtre («[...] un arbre enchanté de minuit, où va venir battre des entrechats, dans un linccul de satin blanc, une svelte trépassée de l'ancienne Comédie-Italienne" II, 379), jardin mémoires (« [...] l'arbre au pied duquel Chateaubriand dormit toute une nuit, la tête des deux Floridiennes sur sa poitrine, l'arbre d'amoureux souvenir, soigné par lui avec tant d'affection dans sa Vallée aux loups [...]" III, 379). Or, après ce déploiement, le texte (et l'ensemble du volume) se referme sur l'évocation du jardin noir, rendu à la nuit et peut-être à la mort, et l'écrivain souligne ainsi que c'est bien lui, par sa description, qui a la maîtrise ultime de ces images : elles ont certes une sorte de vie organique, ici liée au cycle des saisons (ou dépendant, on l'a vu plus haut, du processus de la représentation), mais elles sont surtout, sur fond de vide, en l'absence éventuelle de tout référent (le jardin peut-être " tué par la gelée de cet hiver "), le fruit de l'écriture qui aura su les faire lever puis les effacer.

À l'opposé de La Maison d'un artiste, on pourrait donner l'exemple d'une autre boîte à images, mais surchauffée et déréglée. Dans $A$ rebours (1884), Huysmans s'inspire directement de l'ouvrage d'Edmond de Goncourt ${ }^{17}$, mais son héros procède à une élaboration encore plus rigoureuse de l'espace et multiplie outre mesure les dispositifs producteurs d'images ${ }^{18}$; celles-ci échappent alors à son contrôle et se retournent contre lui. Celles du souvenir cauchemardesque d'une rage de dents (chap. IV), où deux couleurs crues s'imposent (" une dent bleue où pendait du rouge $"$ ), semblent lui faire payer l'excessif raffinement de sa théorie des couleurs (chap. I), augmenté encore par ses spéculations sur les pierreries dont il a paré la tortue chargée d'exalter les teintes de son tapis d'Orient (début du chap. IV). Premier désaveu, la tortue refuse de bouger ; audehors, c'est le noir et le blanc (nuit et neige) qui dominent ; et après la violence

17. D. Grojnowski (éd. Flammarion, GF, 2004) a repéré plusieurs emprunts, qu'on peut encore compléter : la méditation sur la couleur (ch. I) renvoie au choix du rouge chez Goncourt ("Petit salon "); même référence au laqué des " panneaux de voiture " pour la peinture des boiseries; les pièces gigognes de la salle à manger copient les boîtes japonaises du "Cabinet de l'ExtrêmeOrient " (II, 311 sq.), etc.

18. Voir "L'âme des choses: descriptions d'intérieur de Baudelaire à Rodenbach ", dans un volume d'hommages à $P$. Hamon, éd. A. Pagès, à paraître bientôt. 
récurrente de ce rouge sang du rêve, la tortue est retrouvée morte. C'est le moyen de dire que tout ne peut servir à produire des images, et que le vivant particulièrement ne peut être asservi à cette fin. Le corps du héros, trop gavé d'images (à voir, ou - plus complexes encore - à imaginer, par le truchement des autres sens, comme l'odorat), devient à son tour machine à produire des images (mentales), qui dysfonctionne. Une tyrannie de l'image s'empare du sujet impuissant (" [la solitude] guidait un défilé de rêves qu'il subissait, passivement, sans même essayer de s'y soustraire " chap. VII, p. 169), et les images de suppliciés longuement contemplées (la décollation de Jean-Baptiste, les Persécutions de Jan Luyken, le Christ du Gréco) sont la préfiguration de la torture que finiront par lui infliger toutes les images, à commencer par la sienne propre, reflétée par le miroir au sortir de la maladie (chap. XV, p. 330).

Ce qui manque à Des Esseintes, outre une " morale de l'image ", qui l'aurait incité à en user avec modération, c'est surtout la médiation de l'écriture dans la gestion des images. Le seul texte de sa main évoqué dans le roman (l'antienne de Pantin, chap. X) fait resurgir une image stable, et nettement circonscrite, au contraire des enchaînements incongrus du rêve (fin du chap. VIII par exemple) : même s'il semble plongé dans une sorte d'hypnose (" $[\ldots]$ une hallucination l'emporta loin de Fontenay $[. .$.$] ), c'est le retour du même ("le miroir lui$ répercuta en même temps que la rue les réflexions qu'elle avait autrefois fait naître "; "il se répéta cette ingénieuse, mélancolique et consolante antienne qu'il avait jadis notée dès son retour dans Paris "), la récurrence d'une vision fixée par l'écriture, au lieu de l'errance psychique du sommeil.

En dépit du contrôle qu'a su opérer l'écriture sur la prolifération des images, il y en a peut-être quand même encore trop, dans La Maison d'un artiste. Ce que dénonce Huysmans dans le fictif décor d'A rebours reste une dérive possible, un risque latent, non seulement pour l'homme enfermé dans son décor, mais aussi pour l'écrivain cerné par ces images qui le reflètent, et dont il ne sait plus que commander l'apparition ou la disparition.

Cela tient probablement chez Edmond de Goncourt au désir que l'écriture renchérisse sur la saturation matérielle de l'espace réel, empêchant qu'elle se trouve une voie propre. Si l'écriture cherche son autonomie dans l'évocation des images, elle n'en reste pas moins puissamment inféodée chez lui à la transcription du réel, à la fidélité référentielle, en cela doublement tributaire, sur le plan littéraire, d'un réalisme longtemps pratiqué, et sur le plan biographique, d'une collection patiemment constituée. C'est pourquoi Montesquiou souhaitera pour sa part, "pour cette fantaisie de l'écrit d'un hôte à propos de son domicile", une allure "moins collectionneuse que celle du catalogue raisonné de la maison 
d'Auteuil ". Il qualifiera sa propre évocation du "Pavillon des muses", de "Rêverie d'un résident à propos de sa résidence ", se refusant à la description trop détaillée, aux précisions trop érudites : quel que soit le raffinement du décor réel, dans le texte point trop d'images, et suffisamment floues, pour laisser la place à un imaginaire qui n'est peut-être pas de l'ordre du visible... ${ }^{20}$

Dominique PETY

19. Mais la filiation demeure très explicite, avec l'évocation des "reliures au chiffre des Goncourt ", du " profil de bronze de Jules de Goncourt ", " qui décorait naguère le balcon central de La Maison d'un artiste", et la canne royale passée des mains de Goncourt dans les siennes et figurant dans son portrait par Boldini ("Le Pavillon des muses" dans Professionnelles Beautés, Juven, 1905).

20. Voir, à la fin du texte, le choix du merveilleux contre l'érudition historique, ou, en lien direct avec Baudelaire, la finalité ouvertement spirituelle assignée au décor (dans la chambre, un lit qui sert les "élans de l'insatiable, de l'insaisissable Chimère") ; et l'ensemble de l'habitation qui apparaît finalement comme un cadre pour le chant et la musique des vers. 